August 21, 2018 19:36 WSPC/INSTRUCTION FILE iwnd $2009^{\circ}$ trautmann

International Journal of Modern Physics E (C) World Scientific Publishing Company

\title{
THE SYMMETRY ENERGY IN NUCLEAR REACTIONS
}

\author{
W. TRAUTMANN, S. BIANCHIN, A.S. BOTVINA ${ }^{\dagger}$ A. LE FÈVRE, Y. LEIFELS, C. SFIENTI \\ AND THE ALADIN COLLABORATION \\ GSI Helmholtzzentrum für Schwerionenforschung GmbH, \\ Planckstr. 1, D-64291 Darmstadt, Germany \\ N. BUYUKCIZMECI, R. OGUL \\ Department of Physics, University of Selçuk, 42079 Konya, Turkey \\ I.N. MISHUSTIN \\ Frankfurt Institute for Advanced Studies, J.W. Goethe University, \\ D-60438 Frankfurt am Main, Germany \\ M. CHARTIER, P.Z. WU \\ University of Liverpool, Liverpool L69 7ZE United Kingdom \\ R.C. LEMMON \\ STFC Daresbury Laboratory, Warrington, WA4 4AD United Kingdom \\ Q. LI \\ School of Science, Huzhou Teachers College, Huzhou 313000, China \\ J. ŁUKASIK, P. PAWŁOWSKI \\ H. Niewodniczański Institute of Nuclear Physics, Pl-31342 Kraków, Poland \\ A. PAGANO, P. RUSSOTTO \\ INFN-Sezione di Catania and LNS, I-95123 Catania, Italy \\ Received (received date) \\ Revised (revised date)
}

\begin{abstract}
New results for the strength of the symmetry energy are presented which illustrate the complementary aspects encountered in reactions probing nuclear densities below and above saturation. A systematic study of isotopic effects in spectator fragmentation was performed at the ALADIN spectrometer with ${ }^{124} \mathrm{Sn}$ primary and ${ }^{107} \mathrm{Sn}$ and ${ }^{124} \mathrm{La}$ secondary beams of $600 \mathrm{MeV} /$ nucleon incident energy. The analysis within the

\footnotetext{
*w.trautmann@gsi.de

${ }^{\dagger}$ On leave from Inst. for Nuclear Research, Russian Academy of Sciences, 117312 Moscow, Russia

${ }^{\ddagger}$ Present address: Università di Catania and INFN-Sezione di Catania, I-95123, Catania, Italy
} 


\begin{abstract}
Statistical Fragmentation Model shows that the symmetry-term coefficient entering the liquid-drop description of the emerging fragments decreases significantly as the multiplicity of fragments and light particles from the disintegration of the produced spectator systems increases. Higher densities were probed in the FOPI/LAND study of nucleon and light-particle flows in central and mid-peripheral collisions of ${ }^{197} \mathrm{Au}+{ }^{197} \mathrm{Au}$ nuclei at $400 \mathrm{MeV} /$ nucleon incident energy. From the comparison of the measured neutron and hydrogen squeeze-out ratios with predictions of the UrQMD model a moderately soft symmetry term with a density dependence of the potential term proportional to $\left(\rho / \rho_{0}\right)^{\gamma}$ with $\gamma=0.9 \pm 0.3$ is favored.
\end{abstract}

\title{
1. Introduction
}

Microscopic calculations are rather consistent in their predictions for the strength of the symmetry energy at nuclear densities below saturation. ${ }^{1}$ For homogeneous matter, the symmetry energy decreases with decreasing density, roughly in proportion to $\left(\rho / \rho_{0}\right)^{\gamma}$ where $\rho_{0}$ represents the nuclear density at saturation and $\gamma$ is approximately $2 / 3$. The tendency of nuclear matter to cluster modifies this result, however. The symmetry energy is expected to be finite at very low densities, mainly because of $\alpha$-particle formation,, $2[3$ and should reflect the abundance of larger fragments at the freeze-out conditions of multifragmentation reactions. In the Statistical Multifragmentation Model which has been successfully applied to many types of fragmentation reactions, this is accounted for by using, in its standard version, the symmetry-term coefficient of Weizsäcker's formula for stable nuclei for the description of the emerging fragments. ${ }^{4}$ Recent experiments have shown, however, that this scenario of an idealized freeze-out will have to be modified in order to be consistent with isotope distributions and other observables derived from isotopically resolved fragment yields (for references, see reviews $5[6$ and contributions to this proceedings). The excited fragments seem to be modified in the hot environment which has important consequences for astrophysical processes such as the collapse and explosion of massive stars. 7

At higher-than-normal densities, on the other hand, our knowledge regarding the strength of the symmetry energy is much less complete. ${ }^{[56}$ Most of the available results are obtained by extrapolating from below or near saturation to higher densities, thereby relying on a power law parameterization $\left(\rho / \rho_{0}\right)^{\gamma}$ for the density dependence of the potential part of the symmetry term. According to present findings, the density dependence is moderately soft, with $\gamma$ in the range of 0.5 to 1.0 , and appears to be rather consistent among different observables. $\frac{899}{}$ Very recently, a super-soft symmetry term was deduced from the analysis 10 of the charged-pion ratios for ${ }^{197} \mathrm{Au}+{ }^{197} \mathrm{Au}$ reactions at $400 \mathrm{MeV} /$ nucleon incident energy measured by the FOPI collaboration! 11 This result is of particular interest because of its consequences for neutron-star properties (see, e.g., Refs.12[13) but obviously contradicts the extrapolations. To clarify the situation, it will be necessary to employ additional probes sensitive to the high-density stage of the reaction and capable of constraining the model analyses and the deduced parameters of the equation of state of neutron-rich matter. 
The new results presented in the following, obtained from experiments conducted at the GSI laboratory with beams of several hundreds of $\mathrm{MeV}$ per nucleon, address these currently discussed aspects. From an isoscaling analysis of spectator fragmentation, a considerable modification of the symmetry term coefficient representing the fragment properties in the hot freeze-out environment is deduced, and the ratio of neutron versus hydrogen squeeze-outs is shown to be a sensitive observable for determining the symmetry energy at supra-saturation densities.

\section{Isoscaling in Spectator Fragmentation}

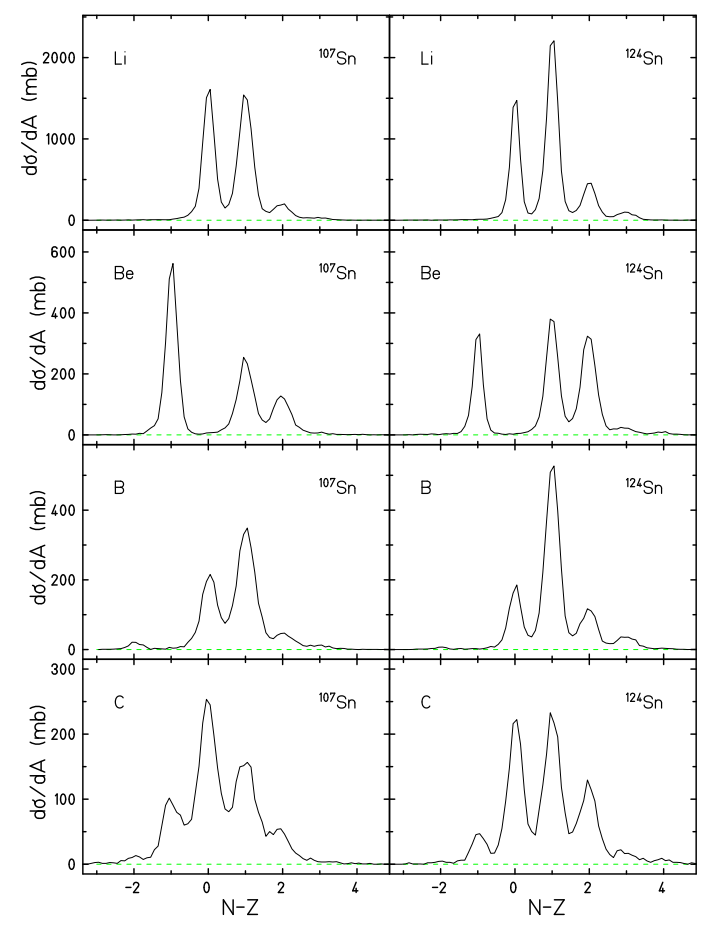

Fig. 1. Inclusive mass distributions for fragments with $3 \leq Z \leq 6$ obtained for ${ }^{107} \mathrm{Sn}$ (left panels) and ${ }^{124} \mathrm{Sn}$ projectiles (right panels).

The ALADIN experiment S254, conducted in 2003 at the SIS heavy-ion synchrotron, was aimed at studying isotopic effects in projectile fragmentation at relativistic energies! ${ }^{14 / 15}$ Besides stable ${ }^{124} \mathrm{Sn}$ beams, neutron-poor secondary ${ }^{107} \mathrm{Sn}$ and ${ }^{124} \mathrm{La}$ beams were used in order to explore a wide range of isotopic compositions. The radioactive beams were produced at the fragment separator FRS 16 by the fragmentation of primary ${ }^{142} \mathrm{Nd}$ projectiles with energies near $900 \mathrm{MeV} /$ nucleon in a thick beryllium target. The FRS was set to select ${ }^{124} \mathrm{La}$ and, in the second part of 
the experiment, ${ }^{107} \mathrm{Sn}$ projectiles which were then delivered to the experiment. All three beams had a laboratory energy of $600 \mathrm{MeV} /$ nucleon and were directed onto ${ }^{n a t} \mathrm{Sn}$ targets with an areal density of $500 \mathrm{mg} / \mathrm{cm}^{2}$. The acceptance for fragments from the projectile-spectator decays was $90 \%$ for fragments with atomic number $Z=3$ and increased gradually to about $95 \%$ for $Z \geq 6$. The obtained mass resolution was $7 \%$ (FWHM) for $Z \leq 3$, decreasing to $3 \%$ for $Z \geq 6$, so that masses for $Z \leq 10$ were individually resolved (Fig. 11).
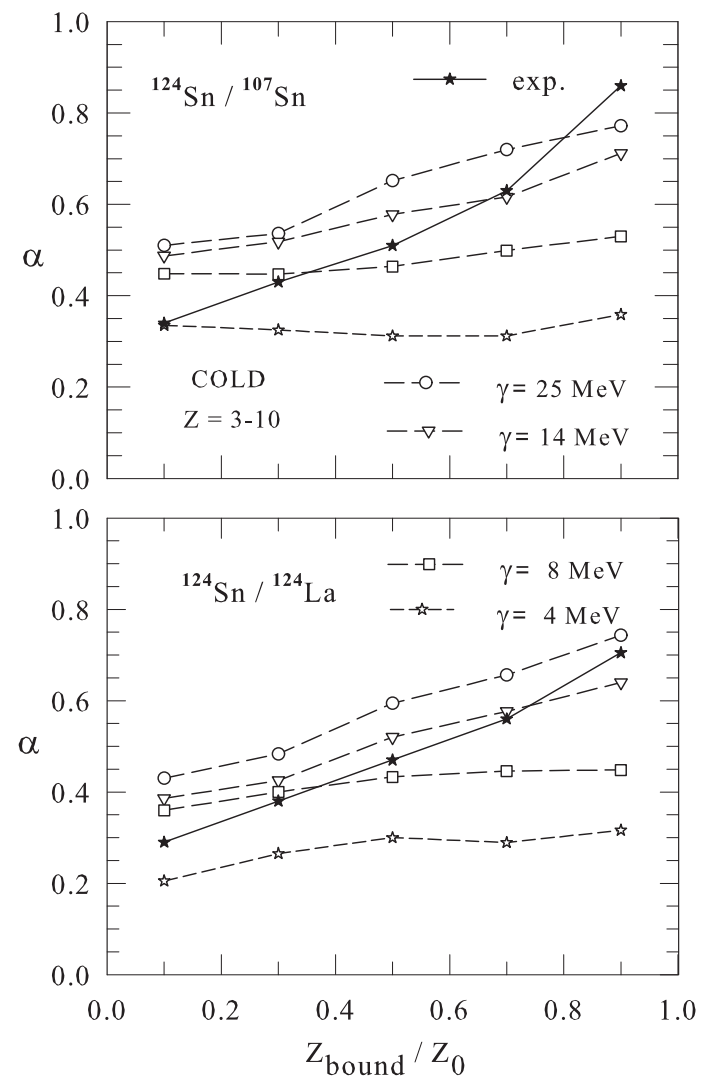

Fig. 2. Experimental data (stars) and SMM ensemble calculations (open symbols) of isoscaling coefficients $\alpha$ extracted from yield ratios of fragments $(3 \leq Z \leq 10)$ from ${ }^{124} \mathrm{Sn}$ and ${ }^{107} \mathrm{Sn}$ projectiles (top panel), and from ${ }^{124} \mathrm{Sn}$ and ${ }^{124} \mathrm{La}$ projectiles (bottom), versus $Z_{\text {bound }}$ normalized with respect to the nominal projectile charges $Z_{0}=50$ and 57 . Four symmetry-term coefficients $\gamma$ were used in the SMM calculations as indicated in the figure.

In order to reach the necessary beam intensity of about 1000 particles/s with the smallest possible mass-to-charge ratio $A / Z$, it was found necessary to accept a distribution of neighbouring nuclides together with the requested ${ }^{124} \mathrm{La}$ or ${ }^{107} \mathrm{Sn}$ 
isotopes. Their mean compositions were $\langle Z\rangle=56.8$ (49.7) and $\langle A / Z\rangle=2.19$ (2.16) for the nominal ${ }^{124} \mathrm{La}\left({ }^{107} \mathrm{Sn}\right)$ beams, respectively ${ }^{17}$ Model studies consistently predict that these $\langle A \mid Z\rangle$ values are also representative for the spectator systems emerging after the initial cascade stage of the reaction. In particular, the differences in $\langle A / Z\rangle$ between the neutron-rich and neutron-poor cases are expected to remain the same within a few percent! $18 \mid 19$

Global fragmentation observables were found to depend only weakly on the isotopic composition! $14[15$ This includes, in particular, the mean multiplicity of intermediate-mass fragments $(3 \leq Z \leq 20)$, the largest atomic number $Z_{\max }$ within a partition, and the evolution of these quantities with $Z_{\text {bound }}$. The sorting variable $Z_{\text {bound }}=\Sigma Z_{i}$ of fragments with $Z_{i} \geq 2$ represents the atomic number $Z$ of the spectator system, apart from emitted hydrogen isotopes, and is inversely correlated with the transferred excitation energy.

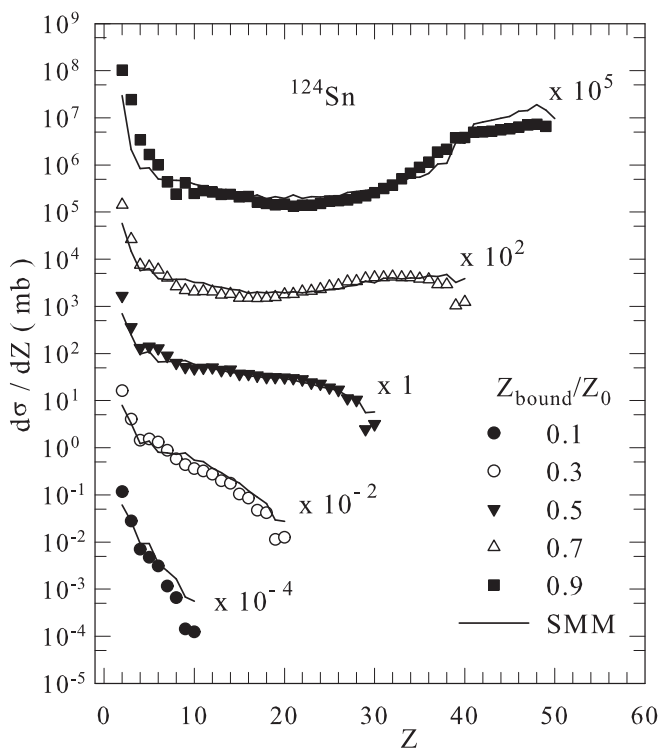

Fig. 3. Measured cross sections for the fragment production in selected $Z_{\text {bound }}$ intervals of 10-unit width as a function of the fragment $Z$ (symbols) in comparison with the results of SMM ensemble calculations (lines) for the case of ${ }^{124} \mathrm{Sn}$ projectiles. The centers of the five $Z_{\text {bound }}$ intervals and the scaling factors used for displaying the cross sections are indicated in the figure.

The comparison of the measured fragment yields from neutron-rich and neutronpoor systems shows that isoscaling is observed 20 The isoscaling parameter $\alpha$, determined from the yields for $3 \leq Z \leq 10$ is found to decrease rapidly as the disintegration of the spectator systems into fragments and light particles increases (Fig. 22), confirming earlier results for the fragmentation of target spectators in re- 
actions of ${ }^{12} \mathrm{C}$ on ${ }^{112,124} \mathrm{Sn}$ at 300 and $600 \mathrm{MeV} /$ nucleon! ${ }^{19}$ Nearly identical results are obtained for the isotopic and isobaric pairs of reactions.

The analysis of the data with the Statistical Multifragmentation Mode 14 was performed with ensemble calculations adapted to the participant-spectator scenario at relativistic energies!21 The ensembles of excited systems with varying excitation energy and mass were chosen so as to best reproduce the charge spectra and correlations observed for the fragment production (Fig. 3). The mean neutron-to-proton ratios $\langle N>/ Z$ for intermediate-mass fragments up to $Z=10$ and the isoscaling parameters were found to be particularly sensitive to the coefficient $\gamma$ of the symmetry term $E_{\text {sym }}(A, Z)=\gamma(A-2 Z)^{2} / A$ in the liquid-drop description of excited fragments at freeze-out. $22 \mid 23$ For the isoscaling parameter $\alpha$, this is demonstrated in Fig. 2. The SMM standard value $\gamma=25 \mathrm{MeV}$ is applicable only in the bin of largest $Z_{\text {bound }}$. Smaller values have to be chosen for reproducing the rapidly decreasing parameter $\alpha$ in the fragmentation regime at smaller $Z_{\mathrm{bound}}$.

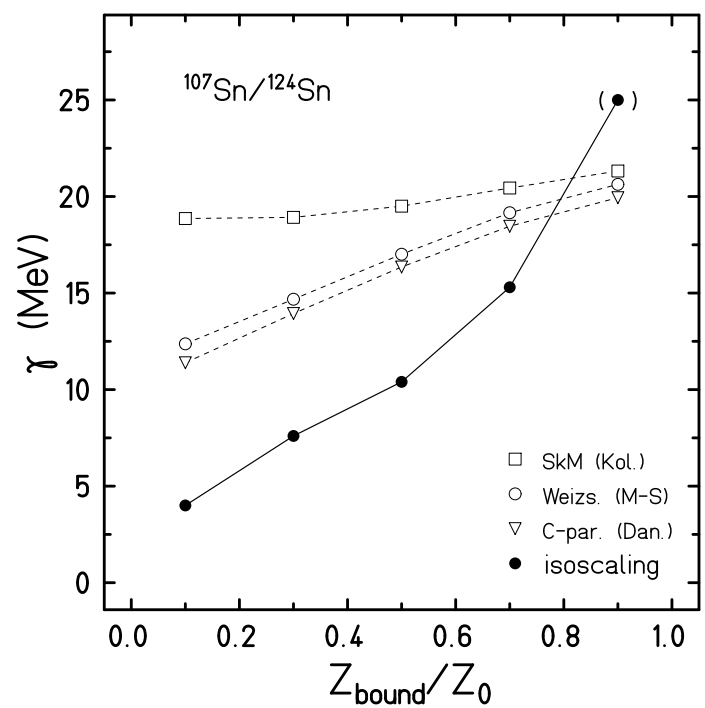

Fig. 4. Symmetry term coefficient $\gamma$ as expected from the changing fragment-mass distributions using surface and volume symmetry-term coefficients from Refs $2425 \mid 26$ (open symbols) in comparison with the coefficient $\gamma$ for hot fragments obtained from the isoscaling analysis for the ${ }^{107,124} \mathrm{Sn}$ pair of reactions with the Statistical Multifragmentation Model (dots).

The symmetry-term coefficient $\gamma$ for hot fragments resulting from the isoscaling analysis for the ${ }^{107,124} \mathrm{Sn}$ pair of reactions is shown in Fig. 4 as a function of $Z_{\text {bound }} / Z_{0}$. For comparison, three predictions are shown in the figure, obtained with rather different approaches but all containing the effect of the surface-symmetry term whose importance increases for the lower-mass fragments. The coefficients of 
the mass formula of Myers and Swiatecki are adapted to ground-state masses.24 From the energies of isobaric analog states, a relation between the volume and surface capacitances of nuclei for absorbing asymmetry $N-Z$ was derived by Danielewicz and Lee, $\stackrel{25}{25}$ while Kolomietz and Sanzhur have used a variational approach using Skyrme forces to derive equilibrium values for the volume symmetry term with surface and curvature corrections for nuclei along the $\beta$-stability line. ${ }^{26}$ With these coefficients, the effective symmetry energy averaged over the set of partitions was calculated for the five bins in $Z_{\text {bound }}$ after the experimental $Z$ distributions had been converted to mass distributions using the projectile $N / Z$. The obtained results show similar trends. The smaller fragments produced at higher excitations cause the effective mean symmetry term to decrease with decreasing $Z_{\text {bound }}$ in all three cases but at a slower rate than that resulting from the isoscaling analysis of the experimental yield ratios.

\section{Neutron and Hydrogen Flows}
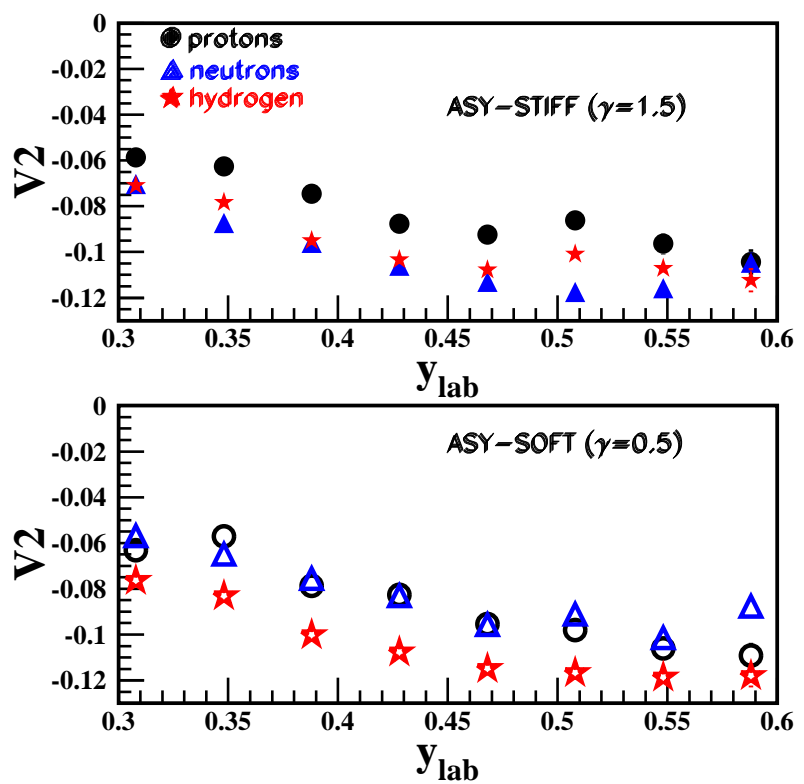

Fig. 5. Elliptic flow parameter $v_{2}$ for mid-peripheral ${ }^{197} \mathrm{Au}+{ }^{197} \mathrm{Au}$ collisions at $400 \mathrm{MeV}$ per nucleon as calculated with the UrQMD model for protons (circles), neutrons (triangles), and the total hydrogen yield (stars) as a function of the laboratory rapidity $y_{\text {lab }}$. The results have been filtered to correspond to the geometrical acceptance of the LAND setup used in the joined experiment. The predictions obtained with a stiff and a soft density dependence of the symmetry term are given in the upper and lower panels, respectively.

In two experiments at GSI combining the LAND and FOPI (Phase 1) detectors, 
neutron and hydrogen collective flow observables from ${ }^{197} \mathrm{Au}+{ }^{197} \mathrm{Au}$ collisions at 400,600 , and $800 \mathrm{MeV} /$ nucleon have been measured $27 / 28$ This data set is presently being reanalyzed in order to determine optimum conditions for a dedicated new experiment, 29 but also with the aim to produce constraints for the symmetry energy at the high densities probed in central collisions at these energies. The results reported here are obtained by comparing with predictions of the UrQMD model which has recently been adapted to heavy ion reactions at intermediate energies. 30

The predictions obtained for the elliptic flow of neutrons, protons, and hydrogen yields for ${ }^{197} \mathrm{Au}+{ }^{197} \mathrm{Au}$ at $400 \mathrm{~A} \mathrm{MeV}$ are shown in Fig. 5. Two values are chosen for the power-law exponent describing the density dependence of the potential part of the symmetry energy, $\gamma=1.5$ (asy-stiff) and $\gamma=0.5$ (asy-soft). The UrQMD outputs have been filtered in order to correspond to the geometrical acceptance of the FOPI/LAND experiment. This produces the asymmetry of $v_{2}$ with respect to mid-rapidity $y_{\text {lab }}=0.448$ because higher transverse momenta are selected with increasing rapidity. The neutron squeeze-out is significantly larger (larger absolute value of $v_{2}$ ) in the asy-stiff case (upper panel) than in the asy-soft case (lower panel) while the proton and hydrogen flows respond only weakly to the variation of $\gamma$ within the chosen interval.

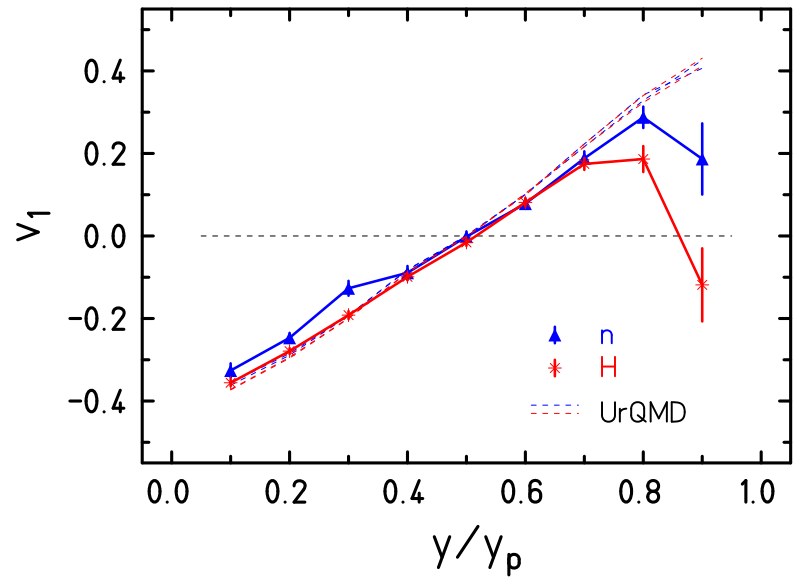

Fig. 6. Directed-flow parameters $v_{1}$ for neutrons (triangles) and hydrogen isotopes (stars) for midperipheral $(5.5<b<7.5 \mathrm{fm})$ collisions of ${ }^{197} \mathrm{Au}+{ }^{197} \mathrm{Au}$ at $400 \mathrm{MeV} /$ nucleon as a function of the scaled rapidity $y / y_{p}$ in comparison with the UrQMD predictions for $\gamma=1.5$ (a-stiff) and $\gamma=0.5$ (a-soft) represented by the dashed lines.

A nearly negligible sensitivity to the stiffness of the symmetry energy is exhibited by the directed flow, according to the UrQMD model. In Fig. 6, the first Fourier coefficient $v_{1}$ of the azimuthal particle distribution with respect to the reconstructed reaction plane is shown as a function of the rapidity $y$, normalized 
with respect to the projectile rapidity $y_{0}=0.896$, and for the interval of impact parameters $5.5 \mathrm{fm}<b<7.5 \mathrm{fm}$. The dashed lines representing the results for neutrons and for hydrogens and for asy-soft and asy-stiff parameterizations of the symmetry energy fall practically on top of each other. The predictions compare well, however, with the experimental results for the multiplicity bin PM3 (see Ref. 27.) expected to correspond to this range of mid-peripheral impact parameters (Fig. 6). The range of rapidities $y \geq 0.8$ at which the data deviate from the expected linearity coincides with the shift of the LAND acceptance to transverse momenta $p_{t} \geq 0.75 \mathrm{GeV} / \mathrm{c} /$ nucleon at which the yields start to drop.

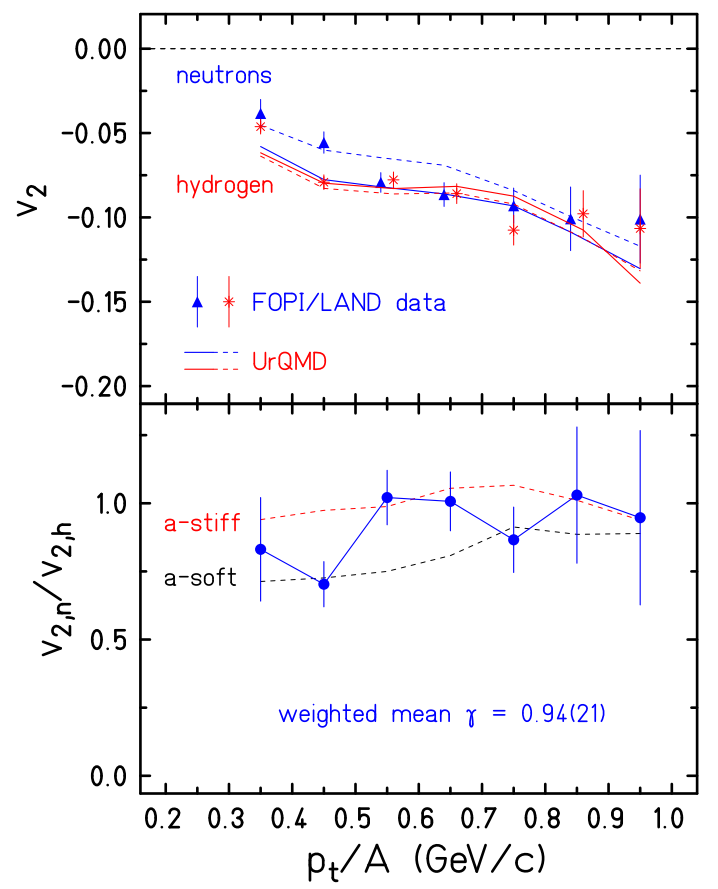

Fig. 7. Elliptic flow parameters $v_{2}$ for neutrons (triangles) and hydrogen isotopes (stars, top panel) and their ratio $v_{2, n} / v_{2, h}$ (bottom panel, dots) for central $\left(b<7.5 \mathrm{fm}\right.$ ) collisions of ${ }^{197} \mathrm{Au}+{ }^{197} \mathrm{Au}$ at $400 \mathrm{MeV} /$ nucleon as a function of the transverse momentum per nucleon $p_{t} / A$ in comparison with the UrQMD predictions for $\gamma=1.5$ (a-stiff) and $\gamma=0.5$ (a-soft) represented by the dashed lines.

The results obtained for the second Fourier coefficient $v_{2}$ describing elliptic flow are shown in Fig. 7 Here the combined data set for central and mid-peripheral collisions (PM3 to PM5) is used and compared to the corresponding UrQMD predictions for $b<7.5 \mathrm{fm}$. The global rise of the absolute magnitude of $v_{2}$ with $p_{t}$ is well described even though the approximately $15 \%$ correction for the dispersion of the reaction plane (cf. Ref. $\frac{31}{\text { }}$ ) is not applied to the data. In contrast to the 
directed flow and as expected from Fig. 5 the squeeze-out of neutrons depends significantly on the symmetry term chosen for the calculations. It is considerably weaker in the asy-soft case while the predictions for the asy-stiff case and for the hydrogen isotopes practically coincide (Fig. 7, upper panel).

For a quantitative evaluation, the ratio of neutron-over-hydrogen flows is proposed as an observable which should be insensitive to uncertainties resulting from the experimental determination of the reaction plane and from the matching of the experimental and theoretical impact-parameter intervals. The comparison shows that the experimental ratios scatter in-between the predictions for the asy-soft and asy-stiff cases (Fig. [7 lower panel). A linear interpolation between these predictions, averaged over $0.3<p_{t} / A \leq 1.0 \mathrm{GeV} / \mathrm{c}$, yields $\gamma=0.94 \pm 0.21$. A smaller but within errors consistent value $\gamma=0.52 \pm 0.30$ is obtained if the comparison is restricted to mid-peripheral impact-parameters $5.5 \leq b<7.5 \mathrm{fm} .32$ The power law coefficients depend weakly on the symmetry energy at saturation chosen in the parameterization. The slight increase of $\gamma$ if this value is lowered confirms that densities above saturation are probed. Other systematic uncertainties have been found to remain within $\Delta \gamma \approx 0.2$. Together with the kinetic term proportional to $\left(\rho / \rho_{0}\right)^{2 / 3}$, the squeeze-out data thus indicate a moderately soft behavior of the symmetry energy at supra-saturation densities.

This result can be considered as, within errors, consistent with the density dependence deduced from fragmentation experiments probing nuclear matter near or below saturation 9 and with the slightly softer density dependence resulting from the analysis of the pygmy dipole resonance in heavy nuclei ${ }^{[8]}$ It also shows that the super-soft density dependence resulting from the IBUU analysis 10 of the $\pi^{-} / \pi^{+}$ yield ratios can, at this time, not be considered as a unique consequence of experiments probing higher densities. Pion yields are expected to be produced during the high-density phase of the reaction $\underline{33}$ but are also subject to considerable in-medium

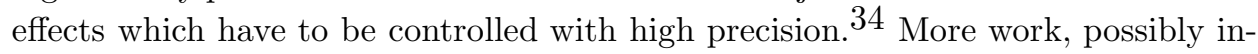
cluding additional observables and focussing on the consistency among them, will be needed in order to arrive at firm conclusions regarding the symmetry energy at high density. In view of the far-reaching and mani-fold consequences, this task deserves highest priority.

Illuminating discussions with Lie-Wen Chen, Bao-An Li, M. Di Toro, and H.H. Wolter are gratefully acknowledged. This work has been supported by the European Community under contract No. HPRI-CT-1999-00001 and FP7-227431 (HadronPhysics2) .

\section{References}

1. M. Baldo, C. Maieron, P. Schuck, X. Viñas, Nucl. Phys. A 736 (2004) 241.

2. C.J. Horowitz and A. Schwenk, Phys. Lett. B 638 (2006) 153.

3. S. Kowalski et al., Phys. Rev. C 75 (2007) 014601.

4. J.P. Bondorf, A.S. Botvina, A.S. Iljinov, I.N. Mishustin, K. Sneppen, Phys. Rep. 257 (1995) 133. 
August $21, \quad 2018 \quad 19: 36 \quad$ WSPC/INSTRUCTION $\quad$ FILE

5. Dynamics and Thermodynamics with Nuclear Degrees of Freedom, ed. by Ph. Chomaz, F. Gulminelli, W. Trautmann, S.J. Yennello (Springer, Berlin/Heidelberg/New York 2006); Eur. Phys. J. A 30 (2006).

6. Bao-An Li, Lie-Wen Chen, Che Ming Ko, Phys. Rep. 464 (2008) 113.

7. A.S. Botvina and I.N. Mishustin, Phys. Lett. B 584 (2004) 233, and preprint arXiv:0811.2593 (2008).

8. A. Klimkiewicz et al., Phys. Rev. C 76 (2007) 051603(R).

9. M.B. Tsang et al., Phys. Rev. Lett. 102 (2009) 122701.

10. Zhigang Xiao, Bao-An Li, Lie-Wen Chen, Gao-Chan Yong, Ming Zhang, Phys. Rev. Lett. 102 (2009) 062502.

11. W. Reisdorf et al., Nucl. Phys. A 781 (2007) 459.

12. M. Kutschera, Phys. Lett. B 340 (1994) 062502.

13. De-Hua Wen, Bao-An Li, Lie-Wen Chen, Phys. Rev. Lett. 103 (2009) 1.

14. W. Trautmann et al., Int. J. Mod. Phys. E 17 (2008) 1838.

15. C. Sfienti et al., Phys. Rev. Lett. 102 (2009) 152701.

16. H. Geissel et al., Nucl. Instr. and Meth. B $\mathbf{7 0}$ (1992) 286.

17. J. Łukasik et al., Nucl. Instr. and Meth. A 587 (2008) 413.

18. A. S. Botvina, O. V. Lozhkin, W. Trautmann, Phys. Rev. C 65 (2002) 044610.

19. A. Le Fèvre et al., Phys. Rev. Lett. 94 (2005) 162701.

20. S. Bianchin et al., in Proceedings of the XLV International Winter Meeting on Nuclear Physics, Bormio, Italy, ed. I. Iori and A. Tarantola, (Ricerca Scientifica ed Educazione Permanente Suppl., vol. 127, Milano, 2007), p. 233.

21. A.S. Botvina et al., Nucl. Phys. A 584 (1995) 737.

22. R. Ogul et al., in preparation.

23. note that the same notation $\gamma$ has been adopted for the symmetry term coefficient of excited fragments in the SMM and for the power-law coefficient describing the density dependence of the symmetry energy.

24. W.D. Myers and W.J. Swiatecki, Nucl. Phys. 81 (1966) 1.

25. P. Danielewicz and J. Lee, Int. J. Mod. Phys. E 18 (2009) 892.

26. V.M. Kolomietz and A.I. Sanzhur, Eur. Phys. J. A 38 (2008) 345

27. Y. Leifels et al., Phys. Rev. Lett. 71 (1993) 963.

28. D. Lambrecht et al., Z. Phys. A 350 (1994) 115.

29. R.C. Lemmon et al., proposal for SIS experiment S394 (2009, unpublished).

30. Qingfeng Li et al., J. Phys. G 31 (2005) 1359.

31. A. Andronic, J. Łukasik, W. Reisdorf, W. Trautmann, Eur. Phys. J. A 30 (2008) 31.

32. W. Trautmann et al., Prog. Part. Nucl. Phys. 62 (2009) 425.

33. M. Zhang et al., Phys. Rev. C 80 (2009) 034616.

34. Jun Xu, Che Ming Ko, Yongseok Oh, preprint arXiv:0906.1602 (2009). 